\title{
Refinamento dapontanasal: Técnica de sutura minimamente invasiva para a ponta nasal larga
}

\author{
Nasal tip narrowing: Minimally invasive suture technique for thick nose tip
}

\section{Antonio Celso Nunes Nassif Filho', Giovana Romano², Denise Braga Ribass ${ }^{3}$, Scheila Maria Gambeta Sass', Emerson Franceschi.}

1) Doutor em Medicina pela UFPR. Professor PUC/PR, Coordenador da Residência Médica em Otorrinolaringologia do Hospital da Cruz Vermelha - Filial do Paraná

2) Título de Especialista em Cirurgia Plástica - AMB / CFM /SBCP. Médica Cirurgiã Plástica.

3) Médica Especializanda em Otorrinolaringologia pelo Hospital da Cruz Vermelha - Filial do Paraná. Residente

4) Especialista em Otorrinolaringologia e Cirurgia Cérvico Facial. Médica Otorrinolaringologista.

5) Médico.

Instituição: Hospital da Cruz Vermelha - Filial do Paraná

Curitiba / PR - Brasil.

Endereço para correspondência: Denise Braga Ribas - Rua Antenor Guimarães, 175 - Casa - Bairro: Pilarzinho - Curitiba / PR - Brasil - CEP: $82110-010$ - Telefone: (+55 41) 9951-0816/3338-1423 - E-mail: deni.ribas@yahoo.com.br

Artigo recebido em 27 de Janeiro de 2011. Artigo aprovado em 19 de Abril de 2011 .

\section{RESUMO}

Introdução: Técnicas de sutura são métodos indispensáveis para esculpir as cartilagens nasais. Para obter um melhor contorno em pontas nasais largas ou globosas os autores apresentam uma técnica de sutura intercrural controlada e minimamente invasiva e os resultados obtidos em 34 pacientes submetidos a rinoplastia.

Objetivo: Demonstrar a técnica de sutura intercrural como tratamento para a ponta nasal larga ou globosa em rinoplastia fechada e apresentar os resultados estéticos.

Método: Estudo retrospectivo de documentos clínicos e fotografias de 34 pacientes ( 5 homens e 29 mulheres), com idade entre 16 e 52 anos, submetidos a rinoplastia com ponta nasal larga ou globosa como elemento anatômico presente no exame físico. Em todos os casos foi utilizada técnica de rinoplastia fechada. Uma sutura inabsorvível é realizada ao longo do processo intermédio. O estudo foi conduzido em 2009 e 2010 no Hospital da Cruz Vermelha Brasileira - Filial do Paraná Brasil e no Hospital Sugisawa. Os pacientes foram operados pelo autor principal e pelos médicos residentes em treinamento.

Resultados: A sutura intercrural controlada proporcionou uma refinamento da ponta nasal e uma melhor forma nos ângulos frontal e lateral da ponta nasal.

Conclusão: A sutura intercrural controlada é um método minimamente invasivo que oferece resultados satisfatórios em refinamento da ponta nasal larga

Palavras-chave: rinoplastia, cartilagens nasais, técnicas de sutura, procedimentos cirúrgicos minimamente invasivos.

\section{SUMMARY}

Introduction: Suture techniques are mandatory methods to shape the nasal cartilages. For purposes of achieving a better contour on thick or globose nasal tips, the authors have approached a controlled intercrural and minimally invasive suture technique and the results obtained by submitting 34 patients to rhinoplasty.

Objective: Illustrate the intercrural suture technique as a treatment for a thick or globose nasal tip under closed rhinoplasty and explain the esthetical results.

Method: Retrospective study with 34 patients' clinical documents and photographs (5 male and 20 female) aged between 16 and 52, who were submitted to the thick or globose nasal tip rhinoplasty as an anatomic element found in the physical examination. In all the cases, closed rhinoplasty technique was used. A suture that cannot be absorbed is performed throughout the intermediate process. The study was performed at the Brazilian Red Cross Hospital in the State of Paraná and Sugisawa Hospital in 2009 and 2010. Patients were operated on by the main author and the junior doctors under training.

Results: The controlled intercrural suture enabled the nasal tip to be narrowed and improved the shape in the frontal and lateral angles of the nasal tip.

Conclusion: Controlled intercrural suture is a minimally invasive method providing the thick nasal tip narrowing with satisfactory results.

Keywords: rhinoplasty, nasal cartilage, suture techniques, minimally invasive surgical procedures. 


\section{INTRODUÇÃO}

As técnicas de refinamento da ponta nasal representam um assunto envolvente na cirurgia de rinoplastia. A satisfação do paciente com o resultado é determinada principalmente pela qualidade do aspecto da ponta nasal no pós-operatório. As primeiras técnicas eram baseadas na remoção de cartilagem, rompimento e liberação ou ainda desestabilização da arquitetura nasal interna. Métodos que rompem os mecanismos de suporte do complexo da ponta nasal $(1,2,3)$.

Após décadas de estudos os conceitos de rinoplastia têm mudado $(1,3,4)$. As ressecções radicais de cartilagem e o rompimento dos mecanismos de suporte têm sido substituídos (5). Utilizam-se técnicas que valorizam a preservação e a reorientação das cartilagens da ponta nasal enquanto mantêm ou restauram os mecanismos intrínsecos de suporte $(1,6,7,8)$.

De 1935 a 1980 a cirurgia da ponta nasal consistiu em excisar, incisar, ou dividir as cartilagens alares $(4,9)$. Os resultados iniciais eram geralmente excelentes, mas hematoma, afinamento dos bordos e colapso de válvula externa ocorriam gradualmente, especialmente sob pele fina (10). A divisão da cartilagem alar foi à falha fatal da técnica, pois leva a perda de suporte e consequentemente resulta em quebras do tecido mole, retração das rimas alares e pinçamento da ponta. Estes narizes, com sua "aparência operada" conduziram a cirurgia nasal à era dos enxertos $(11,12)$.

De qualquer forma, ao lado da inerente variabilidade de enxertos estruturais para contorno, há sempre o risco de extrusão, distorções e de irregularidades aparentes externamente. Para aliviar estas desvantagens dos enxertos, as suturas para modificação da cartilagem alar foram adotadas no final dos anos 80 (13). McCollough e English e Tardy e CHEN, utilizando abordagem endonasal introduziram o conceito de aproximação da ponta com sutura única passando entre 2 cruras intermédias e lateral $(6,8)$.

O objetivo deste estudo é descrever técnica de sutura intercrural controlada em rinoplastia fechada e avaliar o resultado estético do refinamento de pontas nasais largas ou globosas.

\section{MÉTOdO}

Avaliamos as fotos e prontuários de 34 pacientes, 5 homens e 29 mulheres, com idade entre 16 e 52 anos, com ponta nasal larga ou globosa, submetidos a rinoplastia fechada, na qual foi utilizada a técnica de sutura intercrural controlada. As cirurgias foram realizadas no período de janeiro de 2009 a novembro de 2010 no Hospital da Cruz Vermelha Brasileira-Filial do Paraná e noHospital Sugisawa. O trabalho não foi avaliado pelo comitê de ética destes Hospitais.

Todos os pacientes foram submetidos a rinoplastia clássica (técnica básica) de Converse-Diamond, sob anestesia geral e infiltração local (xylocaina 2\% com epinefrina 1:100.000). Todos os pacientes assinaram o termo de consentimento livre e esclarecido.

\section{Descrição da técnica de rinoplastia básica (técnica fechada)}

1. Incisão intercartilaginosa bilateral e liberação das estruturas de tecidos moles do dorso nasal com transfixação septocolumelar.

2. Separação da junção da cartilagem lateral superior do septo nasal com lâmina de bisturi número 15 .

3. Rebaixamento da cartilagem quadrandular.

4. Descolamento do periósteo do dorso nasal.

5. Redução da giba óssea utilizando osteótomo reto (cutting type Maury-Parkes).

6. Septoplastia (quando necessário).

7. Osteotomias laterais realizadas com osteótomo tipo converse $4 \mathrm{~mm}$ curvo. Fratura medial com osteótomo reto.

8. Sutura intercrural controlada realizada para definição de ponta nasal (técnica descrita abaixo).

9. Sutura septocolumelar e sutura da mucosa.

\section{Descrição da técnica de sutura intercrural controlada}

1. Infiltração da mucosa nasal e liberação do epitélio que reveste a cartilagem lateral inferior bilateralmente com tesoura Converse. Com o objetivo de deixar a cartilgem lateral inferior completamente livre do epitélio (Figura 1).

2. Transfixação de cartilagem alar com fio Mononylon 5-0 no seu bordo cranial de no sentido caudal. O local da transfixação da cartilagem pode ser marcado com a compressão digital sobre esta ou através do uso de compasso, para que os pontos sejam simétricos (Figura 2).

Existe a possibilidade de realizar a marcação dos pontos da cartilagem alar a serem transfixados com manobra externa com auxilio de agulhas (insulina) e simulação do efeito da sutura no estreitamento e rotação superior do domus.

3. Passagem da agulha pela incisão septo-columelar para o lado oposto (Figura 3). 


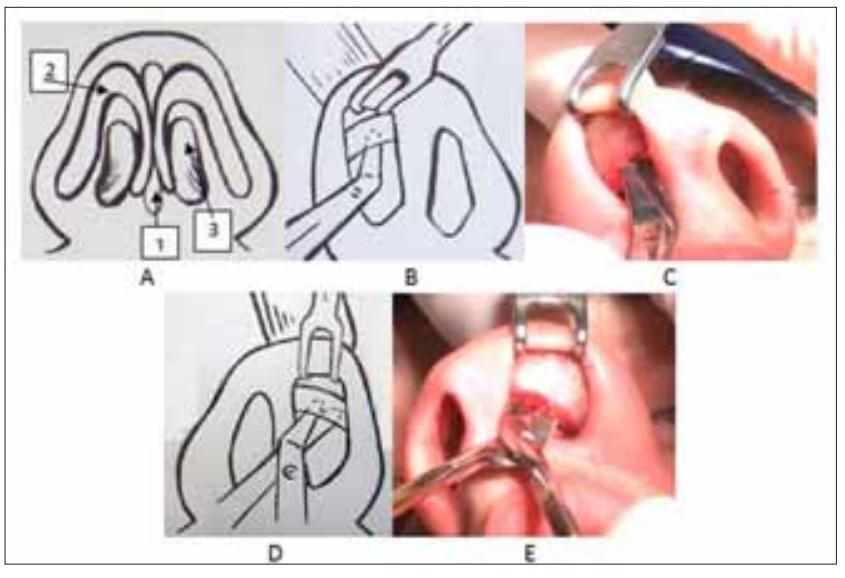

Figura 1. - A: 1 septo nasal, 2 crura lateral, 3 abertura nasal. B, C: Descolamento entre o epitélio e a cartilagem lateral inferior direita com tesoura Converse. D, E: Descolamento cartilagem e a pele lateral esquerda com tesoura Converse

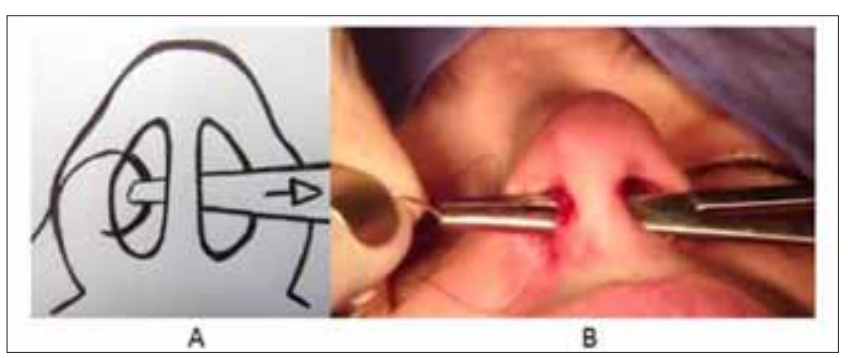

Figura 3. A e B: Passagem do fio mononylon com o auxilio de um porta-agulha da narina direita para a esquerda entre o septo e a columela nasal.

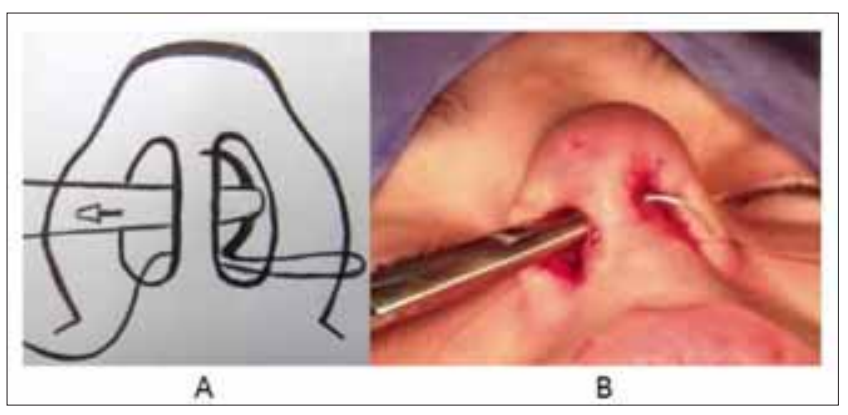

Figura 5. Passagem do fio mononylon com o auxilio de um porta-agulha da narina esquerda para a direita entre o septo e a columela nasal.

4. Transfixação da cartilagem alar no sentido inverso ao da primeira transfixação (Figura 4).

5. Passagem da agulha pela incisão septo columelar para o lado inicial da sutura. (Figura 5).

6. Confecção controlada do nó promovendo a aproximação dos domus de acordo com o refinamento desejado da ponta (Figura 6).

Deve ser realizada a tensão controlada da sutura conforme o aspecto estético observado externamente

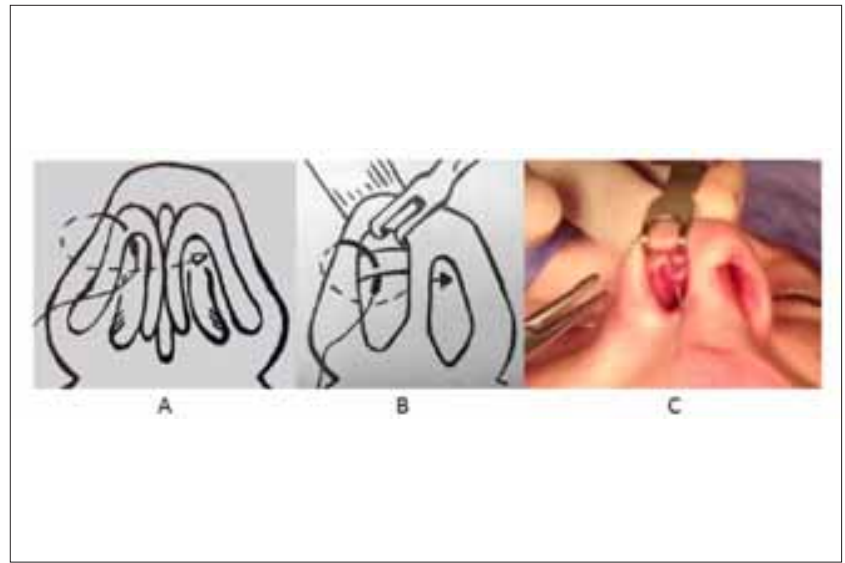

Figura 2. - A, B e C: Realização do ponto na crura lateral direita com fio mononylon 5.0 (observar que o ponto se realiza no sentido caudal).

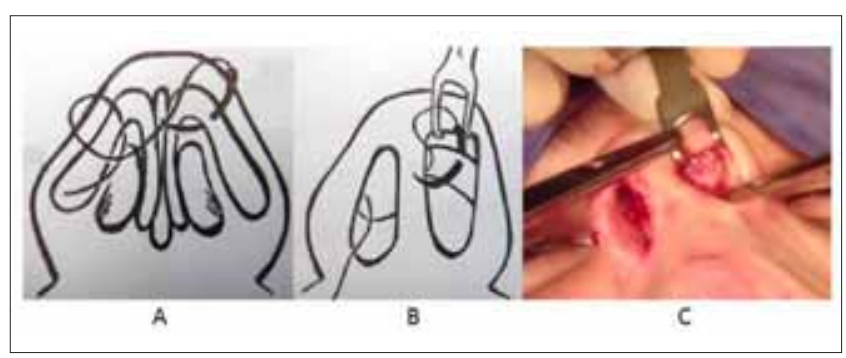

Figura 4. A, B e C: Realização do ponto na crura lateral esquerda com fio mononylon 5.0 no sentido inverso ao ponto realizado do outro lado (cranial).

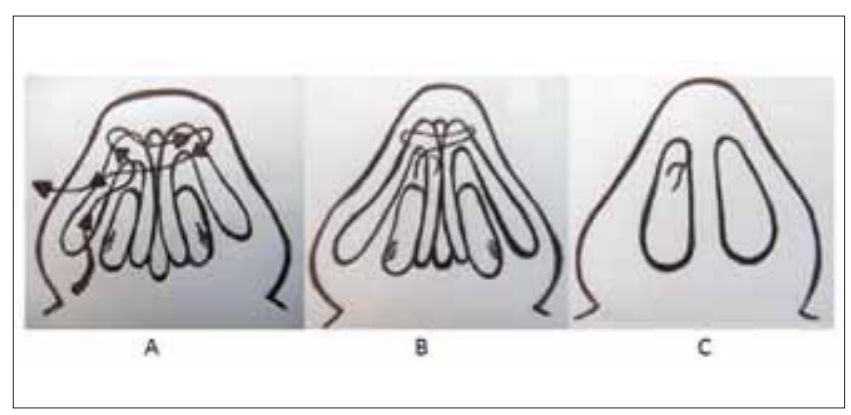

Figura 6. Realização do ponto intercrural.

pelo cirurgião. Se necessário realiza-se mais dois ou mais três nós para fixação da tensão ideal.

\section{RESUlTADOS}

A sutura intercrural controlada foi realizada em 34 pacientes submetidos a rinoplastia fechada. Em $100 \%$ dos casos foi alcançado o estreitamento e a 


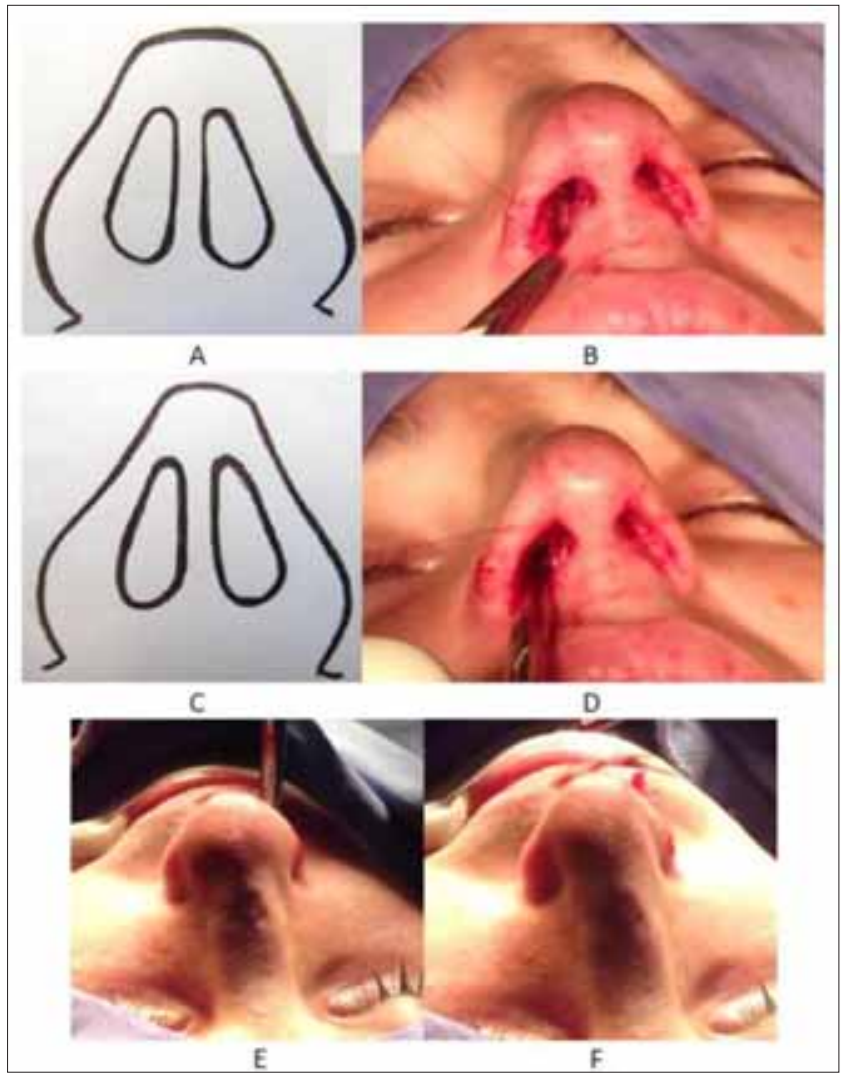

Figura 7. - A e B: Visão da base nasal antes da realização do ponto intercrural (intra-operatório). C e D: Visão da base nasal após da realização do ponto intercrural (intra-operatório). E: Visão superior antes da realização do ponto intercrural (intraoperatório). F: Visão superior após da realização do ponto intercrural (intra-operatório).

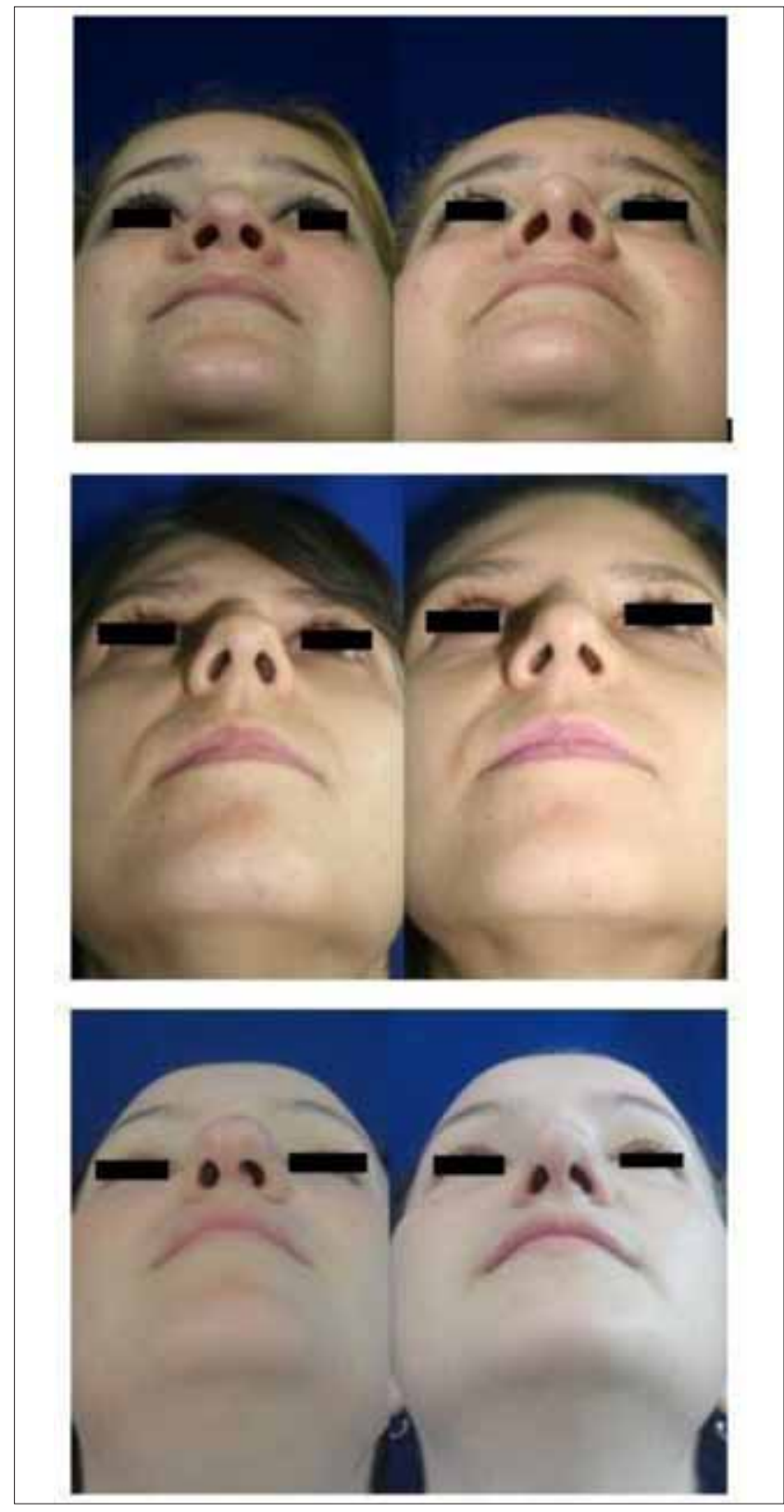

Figura 8. Resultados obtidos com o uso da técnica descrita.

\section{DISCUSSÃO}

definição da ponta nasal bem como melhora do aspecto anterior. O resultado foi satisfatório para o paciente e o cirurgião. As Figuras 7 e 8 comprovam o resultado da técnica descrita no pré-operatório, interoperatório e pós-operatório.

Alguns pacientes se queixaram de um pequeno desconforto na ponta nasal devido à presença do ponto com fio inabsorvível. O ponto foi retirado alguns meses após a cirurgia sem mudança no resultado devido à fibrose cicatricial local.

(a)

.

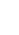

(1)

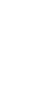

ex 
O controle do contorno da ponta nasal e uma aparência natural da ponta nasal são os as segredos para o sucesso em rinoplastia $(1,15)$. A técnica de sutura intercrural possibilita um refinamento maior ou menor da ponta nasal através da maior ou menor tensão na hora de realizar o ponto entre as cruras laterais.

A técnica de sutura intercrural foi inicialmente descrita, em 1980 por McCollough, Englhishm Tardy e Chen, como uma técnica eficaz e minimamente invasiva (6). Foi o que demonstramos neste artigo, a sutura intercrural pode ser realizada sob rinoplastia fechada um acesso minimamente invasivo.

Por ser uma técnica de refinamento nasal controlado e reversível, a sutura intercrural é um procedimento de primeira escolha na cirurgia da ponta nasal (6). Apesar de não termos demonstrado nenhuma complicação em nossos casos apresentados no artigo, caso houvesse, esta técnica nos possibilita reversão total da ponta nasal como originalmente, o que não ocorre quando se escolhe como primeira opção de tratamento cirúrgico da ponta nasal técnicas mais invasivas.

A convexidade da cartilagem alar é uma das deformidades mais comuns e que requer atenção durante uma rinoplastia (6). A utilização da sutura intercrural possibilita criar um contorno côncavo nesta estrutura.

Das várias técnicas existentes para o afinamento da ponta nasal, a sutura intercrural é a mais indicada quando se opta por rinoplastia fechada e se programa um refinamento sutil da ponta nasal (16).

O resultado final do refinamento nasal é influenciado por fatores como tipo de cartilagem e grau de alargamento da ponta nasal, grau de força intrínseca da cartilagem lateral inferior e força de tensão da sutura promovendo maior ou menor aproximação do domus $(8,17)$.

A espessura da pele é um fator determinante na melhora do contorno na ponta nasal $(12,14)$. Em pacientes com nariz caucasiano o resultado é mais expressivo, pois apresentam pele e cartilagem lateral inferior mais finos $(17,18)$. Em pacientes com pele mais espessa, excesso de tecido subcutâneo e cartilagem lateral inferior mais grossa o efeito da sutura pode ser mais discreto (6).

De acordo com os autores, na técnica de sutura intercrural controlada, devem ser observados alguns detalhes que também foram observado em nosso artigo $(9,15,16)$ :

1. A marcação simétrica dos locais na cartilagem a serem transfixados e aproximados através de manobra externa e simulação do estreitamento.
2. O sentido da realização da sutura para que ocorra rotação superior das cruras laterais e refinamento da ponta nasal.

3. A sutura deve ser realizada com fio não-absorvível.

4. O resultado é menos expressivo em pacientes com pele grossa, cartilagem lateral inferior espessa e presença de excesso de tecido celular subcutâneo acima da cartilagem lateral inferior.

\section{CONCLUSÃO}

A sutura intercrural controlada via rinoplastia fechada é um método efetivo para obtenção de melhor definição da ponta nasal e casos de ponta globosa ou alargada. É uma técnica facilmente aplicável, como demonstrado e ilustrado no método do artigo, que reduz o tempo cirúrgico. A técnica evita a manipulação excessiva dos tecidos cartilaginosos e a presença de cicatriz externa nasal.

Esta técnica representa uma possibilidade de visualização e controle cirúrgico dinâmico do aspecto da ponta nasal, a qual aumenta o arsenal de táticas cirúrgicas para o refinamento do contorno da ponta nasal.

Os resultados obtidos nos 34 pacientes submetidos a esta técnica cirúrgica foi satisfatória tanto do ponto de vista do cirurgião como dos pacientes, a boa qualidade técnica pode ser evidenciada na documentação utilizada nos resultados.

\section{REFERÊNCIAS BIBLIOGRÁFICAS}

1. Behmand RA, Ghavami A, Guyuron B. Nasal tip sutures, part I: the evolution. Plast Reconstr Surg. 2003, 112(4):112529.

2. Tebbetts JB. Shaping and positioning the nasal tip without structural disruption: a new, systematic approach. Plast Reconstr Surg. 1994, 94(1):61-77.

3. Fuleihan NS. The Transvestibular Approach: A new horizon in Rhinoplasty. Arch Facial Plast Surg. 2006, 8(4):27382.

4. Peck GC. Techniques in aesthetic rhinoplasty, $2^{\text {nd }}$ ed. New York: Thieme-Stratton, 1990.

5. McCollough EG, EnglishJL. A new twist in nasal tip surgery: an alternative to the Goldman tip for the wide or bulbous lobule. ArchOtolaryngol. 1985, 111(8):524-9.

6. Baker SR. Suture contouring of the nasal tip. Arch Facial Plast Surg. 2000, 2(1):34-42. 
7. LeachJL, Athré RS. Four suture tip rhinoplasty: a powerful tool for controlling tip dynamics. Otolaryngol Head Neck Surg. 2006, 135(2):227-31.

8. Tardy ME, Cheng E. Transdomal suture refinement of the nasal tip. Facial Plast Surg. 1987, 4(4):317-26.

9. Papel ID. Interlocked transdomal suture technique for the wide interdomal space in rhinoplasty. Arch Facial Plast Surg. 2005, 7(6):414-17.

10. Kridel RW, Konior FJ, Shumrick KA, Wright WK. Advances in nasal tip surgery: the lateral crural steal. Arch Otolaryngol Head Neck Surg. 1989, 115(10):1206-12.

11. Daniel RK. Tip Refinements Grafts: The designer Tip. Aesthet Surg J. 2009, 29(6):528-37.

12. Mocellin M, Pasinato R, Berger CAS, Soares CMC, Grinfeld A, Fagundes MSC. Estreitamento da Base Nasal no Nariz Caucasiano através da técnica de Cerclagem. Arch Otolaryngol. 2010, 4(3):199-205.
13. Daniel RK. Rhinoplasty: creating an aesthetic tip: a preliminary report. Plast Reconstr Surg. 1987, 80(6):77583.

14. Converse JM . Corrective rhinoplasty. In: Converse JM. Reconstructive Plastic Surgery. Philadelphia, Pa WBSaunders Co. 1977, 2:1091-3.

15. Toriumi DM. New Concepts in Nasal Tip Contouring. Arch Facial Plast Surg. 2006, 8(3):156-185.

16. Gruber RP, Friedman GD. Suture algorithm for the broad or bulbous nasal tip. Plast Reconstr Surg. 2002, 110(7):175264.

17. Corrado A, Bloom JD, Becker DG. Domal Stabillization Suture in Tip Rhinoplasty. Arch Facial Plast Surg. 2010, 11(3):194-7.

18. Mocella S, Bianchi N. Double interdomal suture in nasal tip sculpturing. Facial Plast Surg. 1997, 13(3):179-96. 